\title{
Diagnóstico de materiales y lesiones en las fachadas del centro histórico de Cuenca (Ecuador)
}

\author{
María del Cisne Aguirre Ullauri, Edison Maximiliano Castillo Carchipulla y Darío Miguel López \\ León
}

Resumen: Los materiales utilizados en envolventes y fachadas cumplen un papel sustancial en la constitución de los edificios a nivel estético y funcional. La sinergia de agentes climáticos, actividades antrópicas relacionada al uso, intervenciones nocivas, vandalismo y otros factores evidencian deterioro. En el caso del Centro Histórico de Cuenca (Ecuador) este panorama ha sido poco estudiado, por lo que la presente investigación expone una aproximación desde a) el análisis arquitectónico de 35 envolventes, b) la tipificación de lesiones, c) la evaluación de impacto ambiental y, d) la propuesta de aplicación normativa para estudios analíticos previos a la intervención. Se identifican 14 materiales de uso histórico con afecciones en niveles severo y moderado; los más usados y afectados son los cerámicos, térreos y vítreos, además de la madera y el travertino a nivel particular. Se expone la sensibilidad de los materiales frente al ambiente y la demanda de políticas técnicas para su conocimiento y tratamiento. Por su capacidad inferencial se consideran 5 envolventes como el punto de partida para el estudio analítico conforme normativa internacional.

Palabras clave: materiales, lesiones patológicas, aplicación normativa: estudios analíticos, estado de conservación

\section{Material and damage diagnosis on the facades of the Historic Center of Cuenca (Ecuador)}

Abstract: The materials used in architectural envelopes and facades are essential in building construction both, aesthetically and in terms of usefulness. The synergy of climatic agents and human activities related to the use, damaging actions, vandalism, and other issues, evidence their deterioration. In the case of the Historical Center of Cuenca (Ecuador) this panorama has not been amply studied, which is why the present research approaches it from a) the architectural analysis of 35 architectural envelopes, b) the categorization of damages, c) the environmental impact analysis, and d) the normative application proposal for prior intervention analytical studies. This way, 14 historically used materials presenting severe to moderate damages are being identified; the most used and damaged are ceramics, earthen, and glass, as well as wood and travertine in a particular level. It reveals the materials' reaction to the environment and the need for their study and treatment technical policies. Due to their characteristics, five architectural envelopes will be considered as the starting point for the analytical study since international standards.

Keyword: materials, pathological damage, normative application, analytical studies, state of conservation

\section{Diagnóstico de materiais e danos nas fachadas do centro histórico de Cuenca (Equador)}

Resumo: Os materiais utilizados em envolventes e fachadas desempenham um papel essencial na constituição de edifícios a nível estético e funcional. A sinergia de agentes climáticos, atividades antrópicas relacionadas com o uso, intervenções prejudiciais, vandalismo e outros fatores originam deterioração. No caso do centro histórico de Cuenca (Equador), esse panorama foi pouco estudado e por isso esta pesquisa apresenta uma abordagem que envolve: a) análise arquitetónica de 35 envolventes, b) tipificação dos danos, c) avaliação do impacto ambiental e d) proposta de aplicação normativa para estudos analíticos anteriores à intervenção. Identificaram-se 14 materiais de uso histórico com danos severos ou moderados; os mais utilizados e afetados são os cerâmicos, térreos e vítreos, além da madeira e do travertino a um nível particular. Foram expostas a sensibilidade dos materiais ao meio ambiente e a necessidade de políticas técnicas para o seu conhecimento e tratamento. Devido à sua capacidade inferencial, consideraram-se 5 envolventes como ponto de partida para o estudo analítico de acordo com a normativa internacional.

Palavras-chave: materiais, danos patológicos, aplicação normativa, estudos analíticos, estado de conservação 


\section{Introducción}

El Centro Histórico de Cuenca (CHC) situado en la región austral del Ecuador constituye uno de sus asentamientos patrimoniales destacados. Producto de la sucesión histórica entre pueblos prehispánicos e hispánicos, la definición urbana y arquitectónica incluye diversas condiciones de utilización de materiales, sistemas constructivos y conocimientos ancestrales. La arquitectura como el componente tangible más representativo expone problemáticas históricas y tipológicas ampliamente estudiadas, mientras que las asociadas a los materiales, factores y lesiones, son limitadas. El estudio de las últimas tiene aportes específicos en el Atlas de Daños. Edificaciones patrimoniales de Cuenca (Achig Balarezoe et al. 2016) basado en el Monument Damage Diagnosis System desarrollado por KU Leuven y otras universidades europeas (Van Balen y Verstryng 2016) para clasificar lesiones de bienes patrimoniales e ilustrar condiciones semejantes en otras entidades. Su antecedente es el Damage Atlas Historical Cuenca, Ecuador (De Jongh, Van Wijnenddaele, Cardoso y Van Balen 2009). Otro aporte es la Metodología de Manuales de Conservación Preventiva (Cardoso 2009) que identifica y valora afecciones y riesgo. En lo nacional, la contraparte de la administración pública son la Propuesta de mapa de riesgos de bienes patrimoniales inmuebles expuestos a amenazas de origen natural (Rueda 2014), y la Guía de medidas preventivas para amenaza sísmica, seguridad, protección y manejo de bienes culturales (INPC 2011).

Esta falencia superada en escenarios internacionales, no es más que la constante nacional. Los mayores alcances son advertibles y se posicionan en Quito, e incluyen ejercicios de caracterización de materiales (Aguirre Ullauri y Sanz Arauz 2017). En otras ciudades se recurre a estudios arquitectónicos de documentación para contextualizar la relación materiales - lesiones; las últimas si acaso se analizan y evalúan. En el $\mathrm{CHC}$ el diagnóstico patológico es paralelo al relevamiento arquitectónico y como complemento a la actividad proyectual, sin embargo, tampoco alcanza el estudio de la triada material - lesión - factor (es) de incidencia. Por ello, ante los efectos físicos, socio - culturales y ambientales sobre los edificios patrimoniales se plantea el estudio de 35 envolventes desde el análisis arquitectónico y la evaluación de impacto ambiental (EIA).

\section{Antecedentes}

La degradación de la envolvente o fachada de los edificios preocupa reiteradamente; en general es el indicador de la conservación monumental y con frecuencia la causa de las acciones de rehabilitación (Balaras et al. 2005). Las lesiones se agravan con el tiempo; mientras algunas se atenúan, otras se transforman o surgen por cambios antrópicos, generando rechazo social y detrimento del paisaje urbano histórico. Esta condición latente en los centros patrimoniales a escala mundial, también se avista en el CHC que concentra en 224,14 ha actividades de administración, intercambio, vivienda, educación y recreación, y la presión de los desplazamientos transitorios de la ciudad dispersa. Aunque no ha motivado reacciones su acentuación proyecta afecciones en la calidad, durabilidad y funcionalidad (Balaras et al. 2005; Rodrigues, Teixeira y Cardoso 2011).

En 1998 la formulación del expediente para la candidatura de Patrimonio Mundial, identifica la incidencia de los gases de combustión vehicular como una amenaza potencial en la conservación de la arquitectura del CHC (Cardoso 2017: 86). Pero, no es hasta el 2008 que Fundación Natura ${ }^{[1]}$ implementa el primer sistema de seguimiento y diagnóstico ambiental; esta práctica permanente en Quito y Cuenca permite conocer y evaluar la calidad ambiental, sin embargo, no ha logrado medidas conjuntas de conservación. En el caso de los edificios históricos, sensibles al deterioro por uso, abandono, factores ambientales impredecibles, entre otros, los procesos de intervención y mantenimiento también trastocan las propiedades físicas, químicas y mecánicas, en búsqueda de recuperar el dov'era e com'era.

\section{—Fachadas o envolventes: características y gestión en el CHC}

Los materiales recurrentes de las fachadas del $\mathrm{CHC}$ se vinculan a factores culturales. Para Muñoz (2015) la tradición es el aglutinante formal de los edificios populares y, el móvil seguro para la conservación de los rasgos arquitectónicos. Así, la cultura modelada por los factores ambientales, responde a las características funcionales de adaptación conforme el empleo de soluciones constructivas y estilos, y a las fluctuaciones socio económicas, por tanto y según Rodas, Astudillo y Rodas (2018) la transformación de la arquitectura puede ser un indicador en la mejora en la calidad de vida. En este concierto, estilos como el neoclásico y materiales como el mármol, travertino, metales y semejantes, se asumen como la envoltura significativa del CHC. Con ello la noción teórica de fachada, no envolvente, ratifica el soporte efectivo de la forma, función y tecnología.

Entre 1890 y 1940 [2] los ejercicios de proyección arquitectónica tienen connotaciones particulares; la arquitectura de obra nueva se yergue como el modelo de modernización social aunque predominan las adaptaciones. Espinoza y Calle (2002) pioneros en el estudio indican que, por un lado se bordan paramentos exteriores, y por otro se insertan epidermis. En ambos casos se contraponen realidades; la definición histórica progresiva de los modelos arquitectónicos con una inconsciente posibilidad de liberación de la fachada de toda referencia funcional, espacial o estructural. Además, a pesar de que la definición de los recursos teóricos no se conjuga conforme la visión de Albertalli (2009), el cambio entre fachada y envolvente encaja en la dinámica 
edificatoria del CHC. Desde lo vernáculo, popular o colonial y moderno, la tierra, madera, pétreos y fibras vegetales, lo propio evoca la flexibilidad de la noción de envolvente a partir de su versatilidad, es así que, en el proceso de transición, tanto su articulación como desarticulación podría no identificarse.

Al tratarse de edificios preexistentes estas condiciones atribuidas al material y al entorno exponen incidencias sinérgicas sobre la envoltura, denotando deterioro individual y urbano paulatino. Sin embargo, y pese al reconocimiento de esta problemática en el $\mathrm{CHC}$, desde el siglo XVII se exponen casos de intervención monumental que alcanzan relevancia a inicios del siglo XXI sin considerar dicha dimensión, y únicamente enfocados en recuperar las condiciones de ocupación edilicia al tiempo de ennoblecer la envoltura. En ningún caso se inserta como demanda propia para la conservación la actividad de mantenimiento en lo arquitectónico y ambiental, es decir, la gestión de la intervención (Querol 2010). En el caso de bienes públicos, básicamente arquitectura monumental, la gestión supone un despliegue particular de recursos materiales y económicos, pero también excluye el binomio material - lesión. Por su parte, el Plan de Conservación Preventiva articulado al Plan Especial de Centro Histórico del año 2010 pretendía programar acciones de mantenimiento de los bienes patrimoniales y prevenir posibles amenazas, a través de la planificación e intervención profiláctica o paliativa (GAD de Cuenca 2010). Al no concretarse a la fecha, en el camino ordenanzas, reglamentos y normativas con espíritu paliativo han quedado obsoletos. Lo disponible indica que, las edificaciones catalogadas como pertenecientes al Patrimonio Cultural Edificado de la ciudad (VHIAR 1, 2 y 3) [categorías no vigentes en la actualidad], cuyos paramentos frontales sean de materiales vistos (piedra, ladrillo, cerámica, etc.), obligatoriamente deberán mantener sus características naturales, por tanto es prohibido pintarlos, barnizarlos o cubrirlos con cualquier otro material que distorsione su expresión y presentación (Ilustre Consejo Cantonal 2000). Por consiguiente, ante la inexistencia de parámetros técnicos para incluir o tratar materiales, y aunque no se ha constatado de manera fáctica algún nivel de agresión específico ${ }^{[3]}$, el tiempo, ambiente y sociedad, conforman un entorno hostil para la envolvente arquitectónica.

\section{Metodología}

La investigación toma los instrumentos del proyecto Los materiales en el estudio histórico - constructivo ambiental de los conjuntos históricos. El caso de Cuenca, financiado por la Universidad Católica de Cuenca. De tal suerte incluye; a) análisis arquitectónico de la fachada de 35 edificios patrimoniales [figura 1 y 2], b) tipificación de lesiones [figura 4, 6 y 7], c) evaluación de materiales frente al impacto ambiental [tabla 3], y d) propuesta de aplicación normativa para estudios analíticos previos a la intervención [tabla 4]. El primer segmento considera que el Área Histórica y Patrimonial de Cuenca se divide en 178,23 ha de CHC o Área de Primer Orden, así como 30,12 y 15,70 ha de áreas especiales y arqueológicas. En este escenario coexisten 9338 edificaciones, de las cuales un tercio son patrimoniales en diferentes categorías (Heras 2015: 30; Cardoso 2017: 47) [figura 1]. Acometer el estudio de toda el área o los edificios supone una empresa poco factible a corto o mediano plazo, por lo que se incluyen las siguientes variables para definir el alcance concreto:

a) Representatividad patrimonial, es decir, estudiar edificios en todas las categorías de valor, a excepción de las denominadas Sin valor e Impacto negativo, por su significado intrínseco; en el caso de la categoría Emergente, máxima posible, se limita de antemano toda vez que sobrepasa el espectro de intervenciones o análisis convencionales. Por tanto, las categorías de interés son Valor Arquitectónico A (VAR A), Valor Arquitectónico B (VAR $B$ ) y Valor Ambiental (VA), como elementos de definición temporal ${ }^{[4]}$ y significancia histórica en la urbe ${ }^{[5]}$, al incluir la diversidad de materiales característicos de la práctica arquitectónica, y propender a la evaluación inferencial

b) Representatividad en la definición histórica de la ciudad, al considerar el $\mathrm{CHC}$ como el núcleo fundacional y polifuncional, el conjunto de edificaciones a él asociadas representa desde las instancias estilísticas y constructivas el primer espacio de interés. Desde él, la definición progresiva de la ciudad ha dado lugar a situaciones que, en lo material y su relación con el ambiente, son desconocidas. Bajo esta consideración los casos de estudio promueven la definición de un marco de referencia para proyectar acciones de conocimiento científico desde la aplicación de normativas internacionales. Incluye como variables específicas de selección:

1. 1. Localización en el Área de Primer Orden conforme las ordenanzas (1983 y 2010) y desde las declaratorias nacional (1982) y mundial (1999).

2. Representatividad en área y uso de materiales.

3. Uso histórico y contemporáneo de materiales.

c) Representatividad en el contexto inmediato, es decir, relevancia en términos de emplazamiento, ya sea en tramo, eje vial o barrio [figura 2]. Además, se considera que:

1. El caso seleccionado no necesariamente corresponde al edificio de mayor escala, sino a aquel de condiciones materiales concomitantes con la práctica arquitectónica de Cuenca.

2. La selección corresponde a ejemplos que eventualmente han sido estudiados por su relevancia individual y por tanto sería factible contrastar información.

3. La selección corresponde a edificios que definen la identidad material en su contexto inmediato, ya sea por la armonía con el conjunto, o relación arquitectónica, urbana y paisajística. 


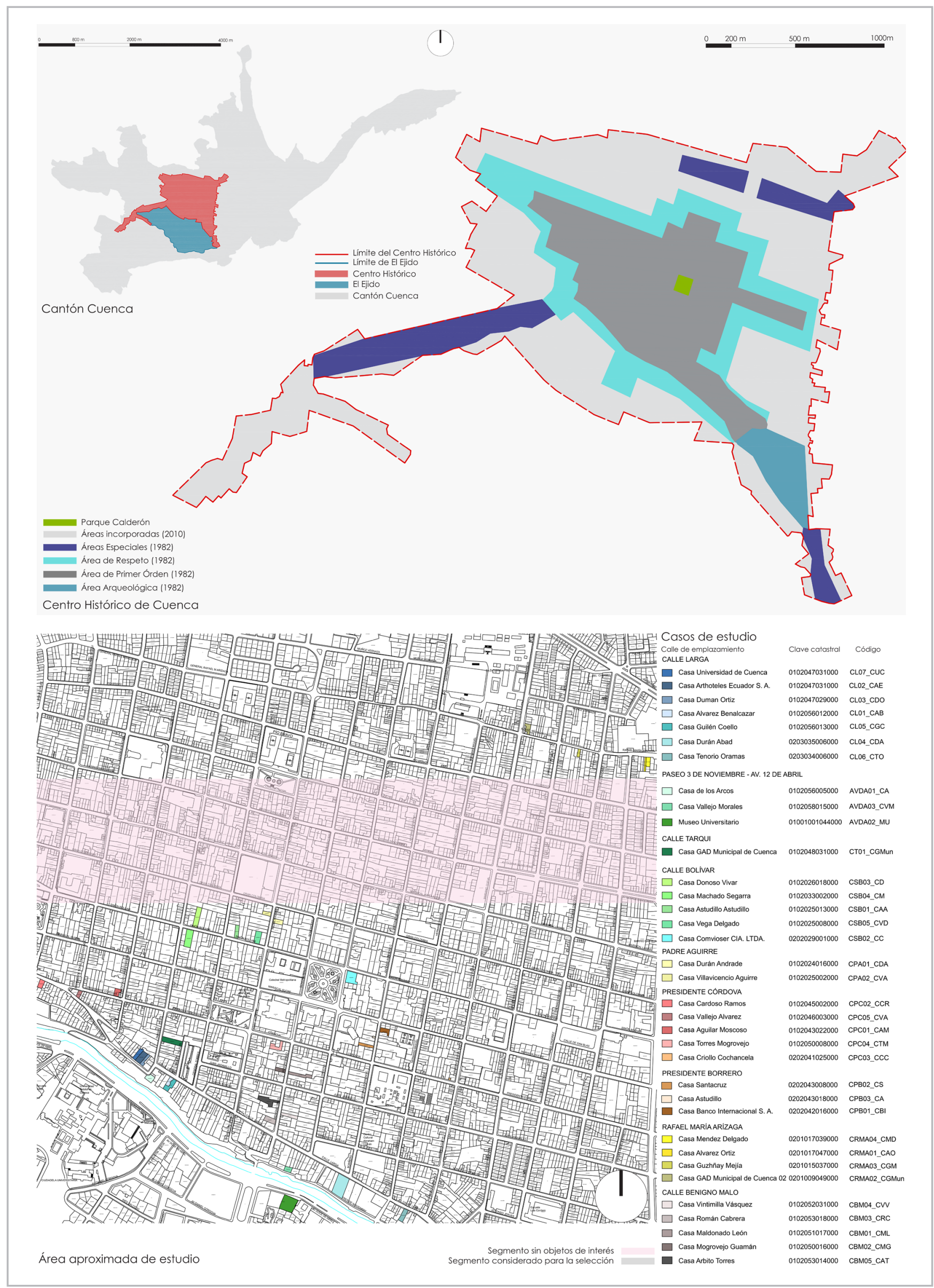

Figura 1.- Cartografía de los casos de estudio. Fuente: Dirección de Áreas Históricas y Patrimoniales 2017. Elaboración: Aguirre Ullauri 2019. 


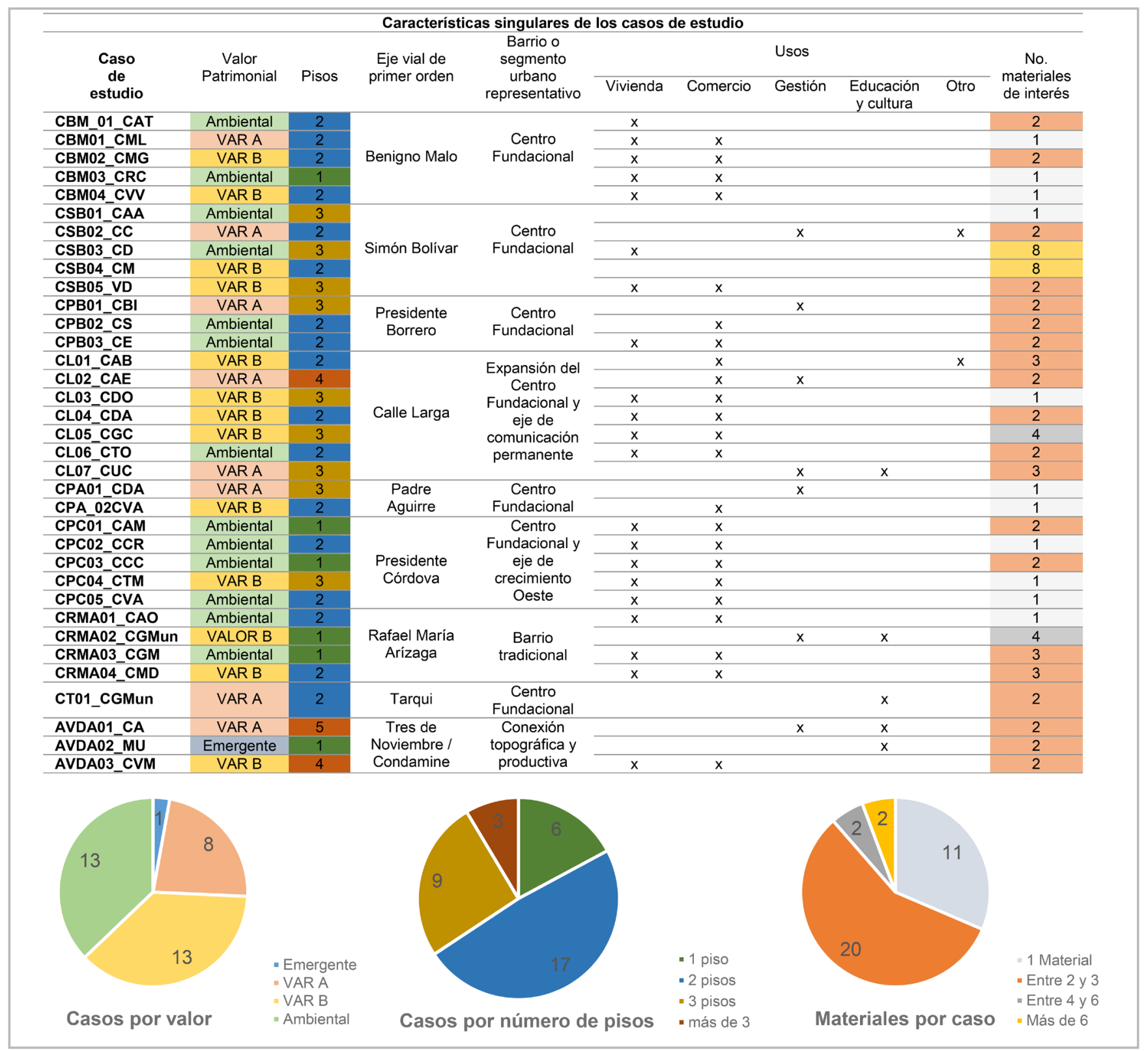

Figura 2.- Características de los casos de estudio. Fuente: Aguirre Ullauri et al. 2018. Elaboración: Aguirre Ullauri 2019

La estrategia para la selección de casos busca reconocer a precisión la importancia de las edificaciones, cuya tendencia a pasar desapercibidos es frecuente, sin embargo, constituyen segmentos de relevancia a la inmutabilidad y/o transformación de la ciudad y el paisaje urbano histórico. Así se orienta a aquellas que en el marco de los literales a), b) y c), representen formas tradicionales de ocupación y por tanto permiten mantener la vitalidad citadina. En relación a los parámetros de exclusión del literal a), no se analizan obras nuevas, arquitectura del movimiento moderno o contemporáneo, culto y asistencia social. Tampoco se considera la existencia o no de intervenciones potencialmente nocivas.

Seguidamente, el levantamiento arquitectónico y fotográfico asistido por aeronaves no tripuladas de las fachadas y la aplicación de las fichas técnico - documentales elaboradas por Aguirre Ullauri, Solano Peláez, García, López, Carrión y Yamunaqué (2018) permiten identificar materiales, sistema constructivos, características organolépticas y lesiones patológicas según Rodríguez (2004) [figura 3].

Finalmente, la propuesta analítica de aplicación normativa y caracterización de materiales toma como base la EIA definido por Aguirre Ullauri, Solano Peláez, Segarra Villacís y Carrión Cabrera (2018); esto es 1) identificar interacciones entre materiales y factores del medio, así como la actividad antropogénica y el estado de conservación del edificio, 2) cuantificar y cualificar la importancia de las interacciones y, 3) determinar la EIA [figura 4]. Con ello se orienta el proceso de estudios analíticos desde el contexto europeo, estadounidense y peruano. 


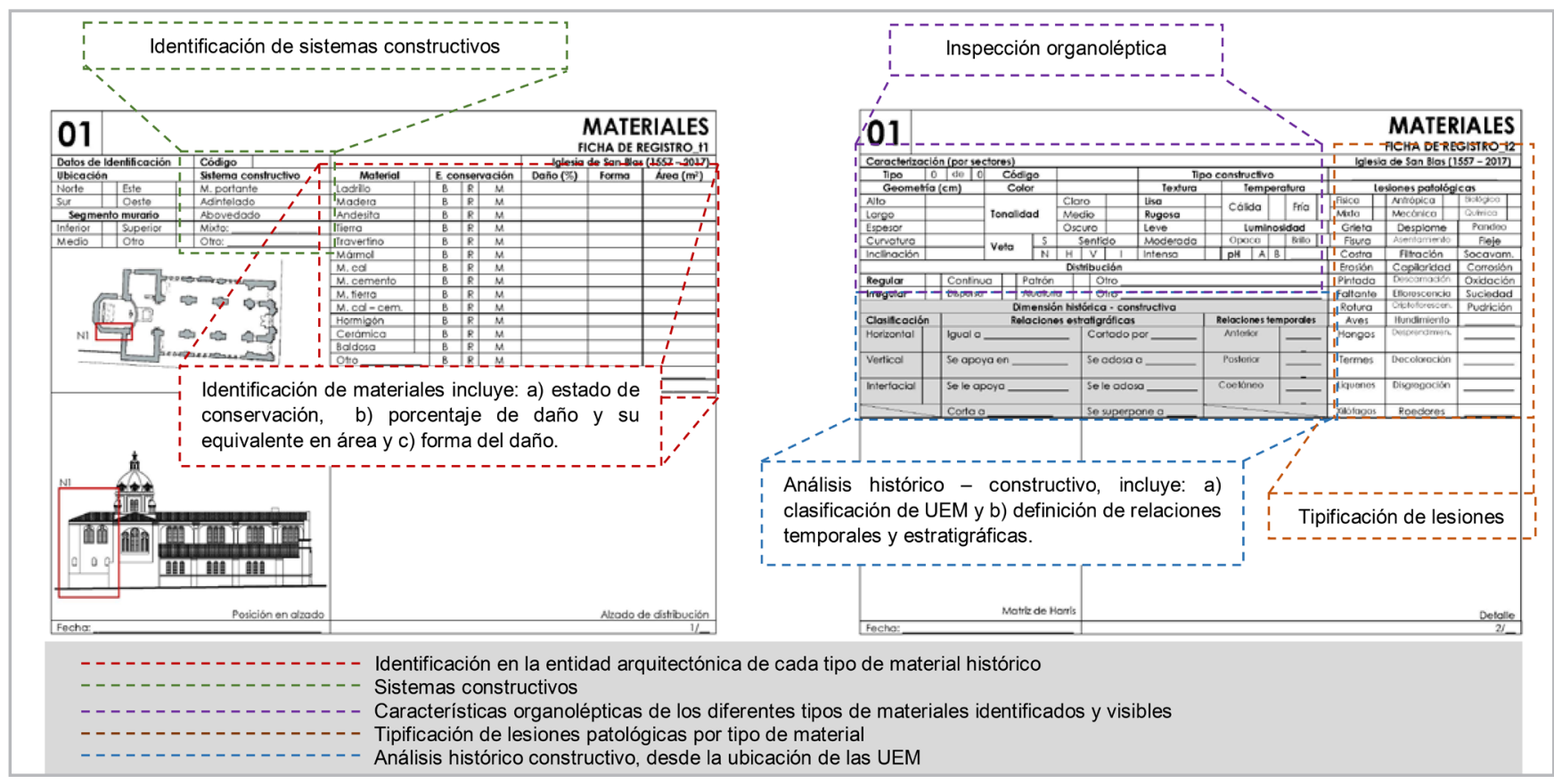

Figura 3.- Modelo de ficha de levantamiento arquitectónico. Fuente: Aguirre Ullauri et al. (2018). Elaboración: Aguirre Ullauri 2019

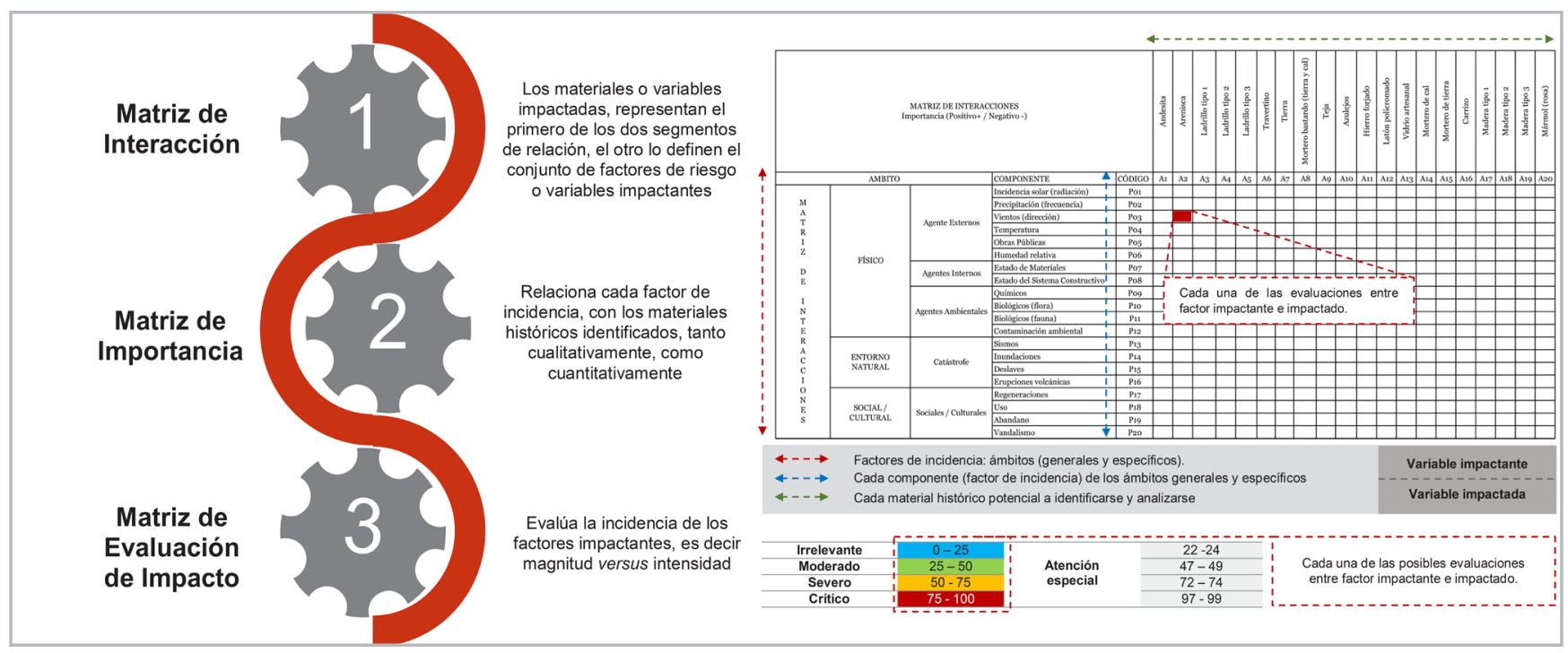

Figura 4.- Metodología de ElA. Fuente: Aguirre Ullauri et al. 2018. Elaboración: Aguirre Ullauri 2019

\section{Resultados y discusión}

Sobre el levantamiento de información se determina que en las 35 fachadas se depositan 14 de 20 materiales definibles como históricos [tabla 1]. Es decir, no se estudian azulejos, teja, vidrio artesanal, carrizo, arenisca y latón policromado. En casos como el ladrillo y la madera se reconocen al menos tres variaciones, según aparejo, geometría y características organolépticas [figura 6]. Esta situación evidencia la inexistencia de insumos técnicos de discriminación de materiales, y posiciona como escenario investigativo a la identificación de especies y los estudios de mensiocronología, arqueometría y cronotipología ${ }^{[6]}$.
Otros segmentos de interés es el comportamiento de los materiales frente a la radiación y presencia de agua, variaciones térmicas, de humedad relativa, entre otros.

Los cerámicos, térreos y vidrios predominan, aunque también reportan representatividad pétreos como el travertino. El resto de materiales tienen uso menor al promedio [figura 5]. Consecuentemente, la lógica edificatoria indica que el sistema constructivo portante prevalece, ya sea a partir de tierra, ladrillo o estructuras mixtas lineales o abovedadas. También se ratifica al adobe como soporte por excelencia de las envolventes del $\mathrm{CHC}$ (Achig Balarezo et al 2013). 


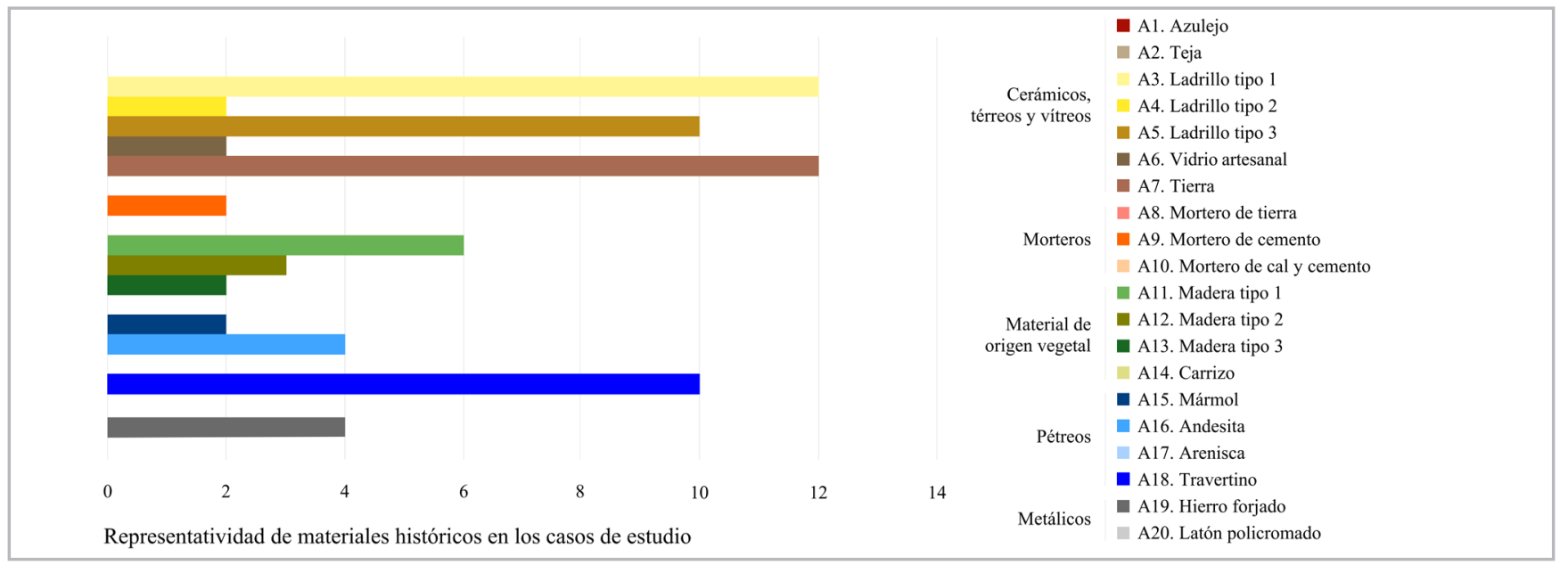

Figura 5.- Representatividad por material. Fuente y Elaboración: Autores

\begin{tabular}{|c|c|c|c|c|c|c|c|c|}
\hline \multicolumn{9}{|c|}{ Características organolépticas de materiales constructivos } \\
\hline Clasificación & Cód. & Material & Untuosidad & Color & Tono & Luminosidad & Textura & Rugosidad \\
\hline \multirow{7}{*}{$\begin{array}{c}\text { Cerámicos, } \\
\text { térreos y } \\
\text { vítreos }\end{array}$} & $\mathrm{A} 01$ & Azulejo & nula & verde & claro & brillante & lisa & nula \\
\hline & $\mathrm{A} 02$ & Teja & nula & anaranjado & medio & opaco & lisa & nula \\
\hline & $\mathrm{A} 03$ & Ladrillo tipo 1 & nula & anaranjado & claro & opaco & rugosa & leve \\
\hline & A04 & Ladrillo tipo 2 & nula & anaranjado & medio & opaco & rugosa & leve \\
\hline & A05 & Ladrillo tipo 3 & nula & anaranjado & mixto & opaco & rugosa & media \\
\hline & A06 & Vidrio artesanal & nula & transparente & claro & brillante & lisa & nula \\
\hline & A07 & Tierra & leve & café & oscuro & opaco & rugosa & leve \\
\hline \multirow{3}{*}{ Morteros } & A08 & Mortero de tierra & leve & café & oscuro & opaco & rugosa & nula \\
\hline & A09 & Mortero de cal & leve & blanco & claro & opaco & rugosa & leve \\
\hline & A10 & Mortero cal-cemento & leve & gris & claro & opaco & rugosa & leve \\
\hline \multirow{4}{*}{$\begin{array}{l}\text { Material de } \\
\text { origen } \\
\text { vegetal }\end{array}$} & A11 & Madera tipo 1 & nula & marrón & claro & opaco & lisa & nula \\
\hline & A12 & Madera tipo 2 & nula & marrón & medio & opaco & lisa & nula \\
\hline & A13 & Madera tipo 3 & nula & marrón & oscuro & opaco & lisa & nula \\
\hline & A14 & Carrizo & nula & mostaza & claro & brillante & lisa & nula \\
\hline \multirow{4}{*}{ Pétreos } & A15 & Mármol & nula & blanco & claro & brillante & lisa & nula \\
\hline & A16 & Andesita & nula & gris & oscuro & opaco & lisa & nula \\
\hline & A17 & Arenisca & nula & gris & claro & opaco & lisa & nula \\
\hline & A18 & Travertino & nula & rosado & claro & brillante & lisa & leve \\
\hline \multirow{2}{*}{ Metálicos } & A19 & Hierro forjado & nula & negro & oscuro & opaco & lisa & nula \\
\hline & A20 & Latón policromado & nula & policromía & medio & brillante & rugosa & media \\
\hline & & Simbología & Materiale & nalizados & & Materiales no & alizados & \\
\hline
\end{tabular}

Tabla 1.- Características organolépticas. Fuente y Elaboración: Autores

Las características organolépticas como aproximación al material sin ayuda de instrumentos de medición evidencian que, tanto el acabado como el color, las variaciones de tono o la untuosidad constituyen escenarios propicios para el deterioro progresivo o particularmente agresivo del medio, más cuando convergen [tabla 1]. Las prácticas locales de obtención, transporte, comercialización y coexistencia sincrónica en el edificio, las condicionan. De ello el mantenimiento y/o intervención juegan un segmento de relevancia para reconocer el impacto ambiental a través de los síntomas del proceso patológico. Además, exponen la inmediatez correctiva y su contraproducente acción catalizadora de síntomas y cambios cualitativos (Douglas-Jones, Hughes y Yarrow 2016). En el último escenario, bajo orientaciones mixtificadas entre el Restauro Estilístico y la Notoriedad, prácticas típicas de la urbe, la esencia material e histórica entendida como proceso natural en la edificación refleja neofilia (Lorenz 2004). Por tanto, la evidencia del paso del tiempo como determinante de la transformación no tiene cabida, y deriva en estructuras arquitectónicas atemporales y vulnerables al deterioro por incompatibilidades; puede entonces pendular el deterioro entre la degradación total y el ennoblecimiento superlativo con todos sus matices (Sahady Villanueva y Gallardo Gastelo 2009). En ambientes como el CHC se acentúa, al reflejar las condiciones más pendencieras de la urbe (Orellana Samaniego, Sellers Walden y Martínez Gavilánez 2017).

En el campo de las lesiones patológicas si bien no se puede hablar de un patrón de comportamiento físicos o temporales, ante la inexistencia de datos previos, se determina que las características organolépticas más 
desfavorables elevan la vulnerabilidad del material al medio, pero a su vez facilitan la visibilidad de la lesión [tabla 1]. En los casos, el deterioro físico (desprendimientos, suciedad por depósito y humedad), antrópico (grafitis) y estructural (fisuras y grietas) describe el cuadro patológico general [figura 7]. Lo propio se ratifica desde el análisis por material [figura 8]. A excepción del mortero de tierra, los materiales presentan suciedad por depósito y grafitis como incidencias alarmantes; mientras que las fisuras, grietas y humedad sin llegar a ser despreciables, son menos significativas. Por su parte, las afecciones químicas no son representativas a simple vista, no así el estado de conservación de materiales como el hierro forjado, colado o fundido.

Sobre la descripción general y desde la EIA [tabla 2] se confirma que los materiales más afectados son la tierra, ladrillo, hierro forjado y madera, así también que las condiciones ambientales reportan incidencias de corrección y mitigación, que se corresponden con los niveles Severo y Crítico de la Matriz de Leopold [figura 4]. Adicionalmente, el nivel de Atención Especial toma protagonismo como complemento para orientar las prioridades de intervención, ya que supone vulnerabilidad potencial ante la agresividad del ambiente.

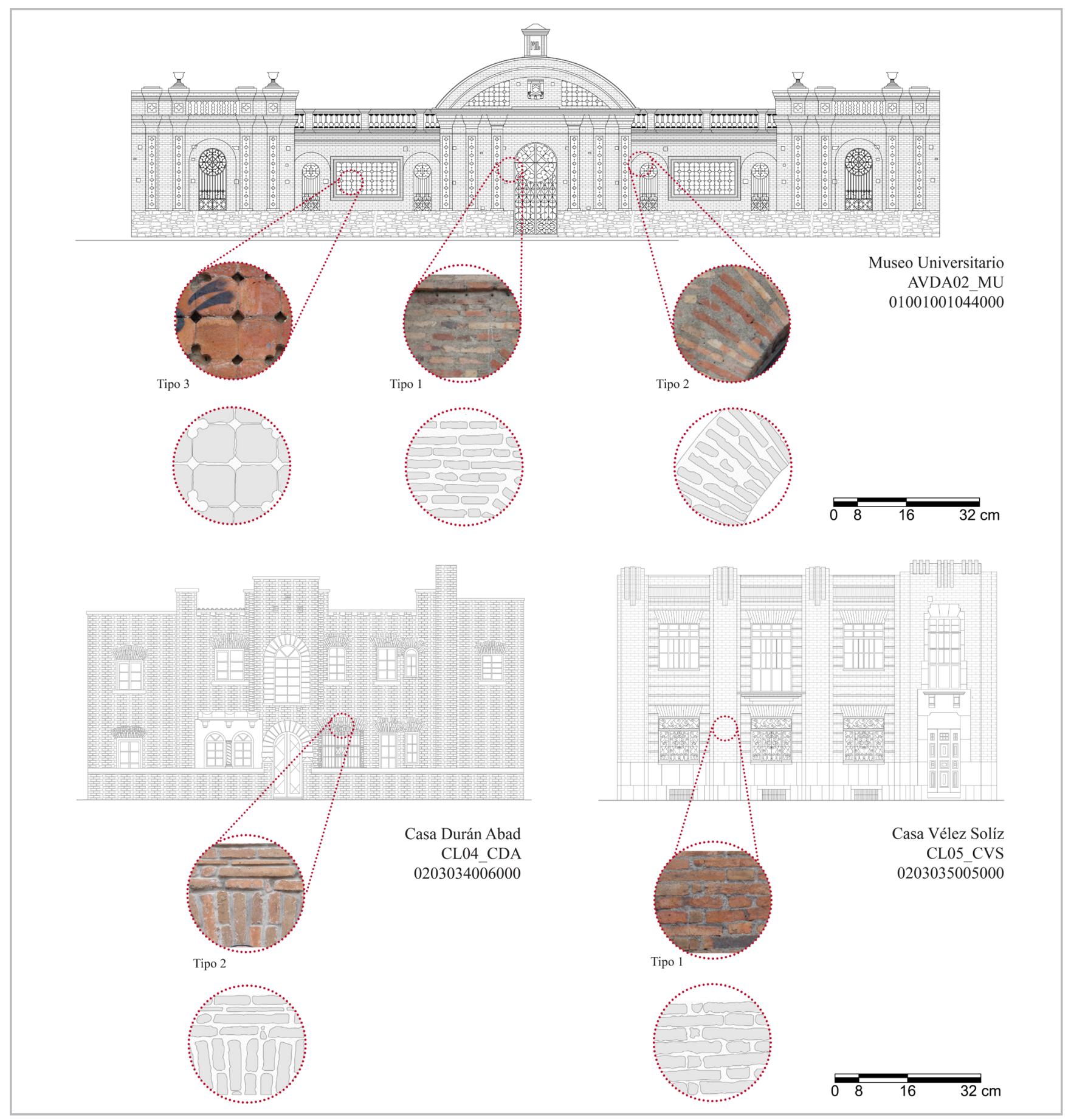

Figura 6.- Tipos de ladrillos: reconocimiento preliminar. Fuente y Elaboración: Autores 


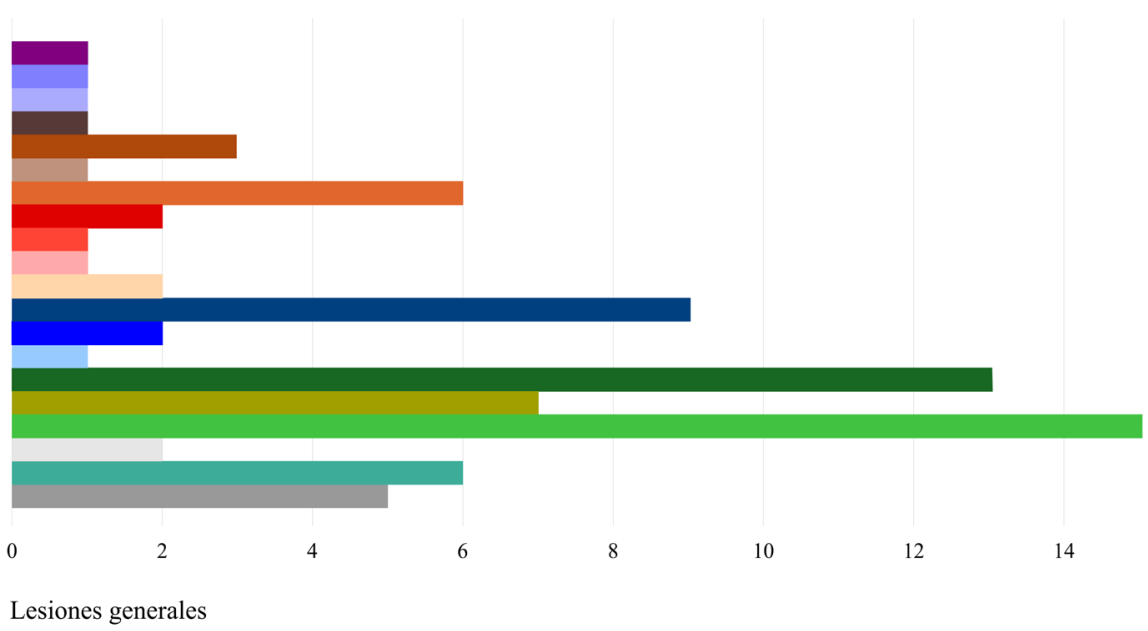

$\begin{aligned} & \square \text { Oxidación } \\ \text { Química } & \square \text { Eflorescencias } \\ & \square \text { Meteorización } \\ & \square \text { Disgregación } \\ & \square \text { Agrietamiento } \\ \text { Mecánica } & \square \text { Deformación } \\ & \square \text { Fisuración } \\ & \square \text { Colonización por líquenes } \\ \text { Biológica } & \square \text { Presencia y acción de aves } \\ & \square \text { Pudición parda } \\ & \square \text { Pudrición blanca } \\ & \square \text { Graffiti } \\ \text { Antrópica } & \square \text { Pintura } \\ & \square \text { Cambio de material } \\ & \square \text { Humedad } \\ & \square \text { Suciedad por depósito } \\ \text { Física } & \square \text { Desprendimiento } \\ & \square \text { Erosión } \\ & \square \text { Descamación } \\ & \square \text { Descascaramiento }\end{aligned}$

Figura 7.- Lesiones generales. Fuente y Elaboración: Autores

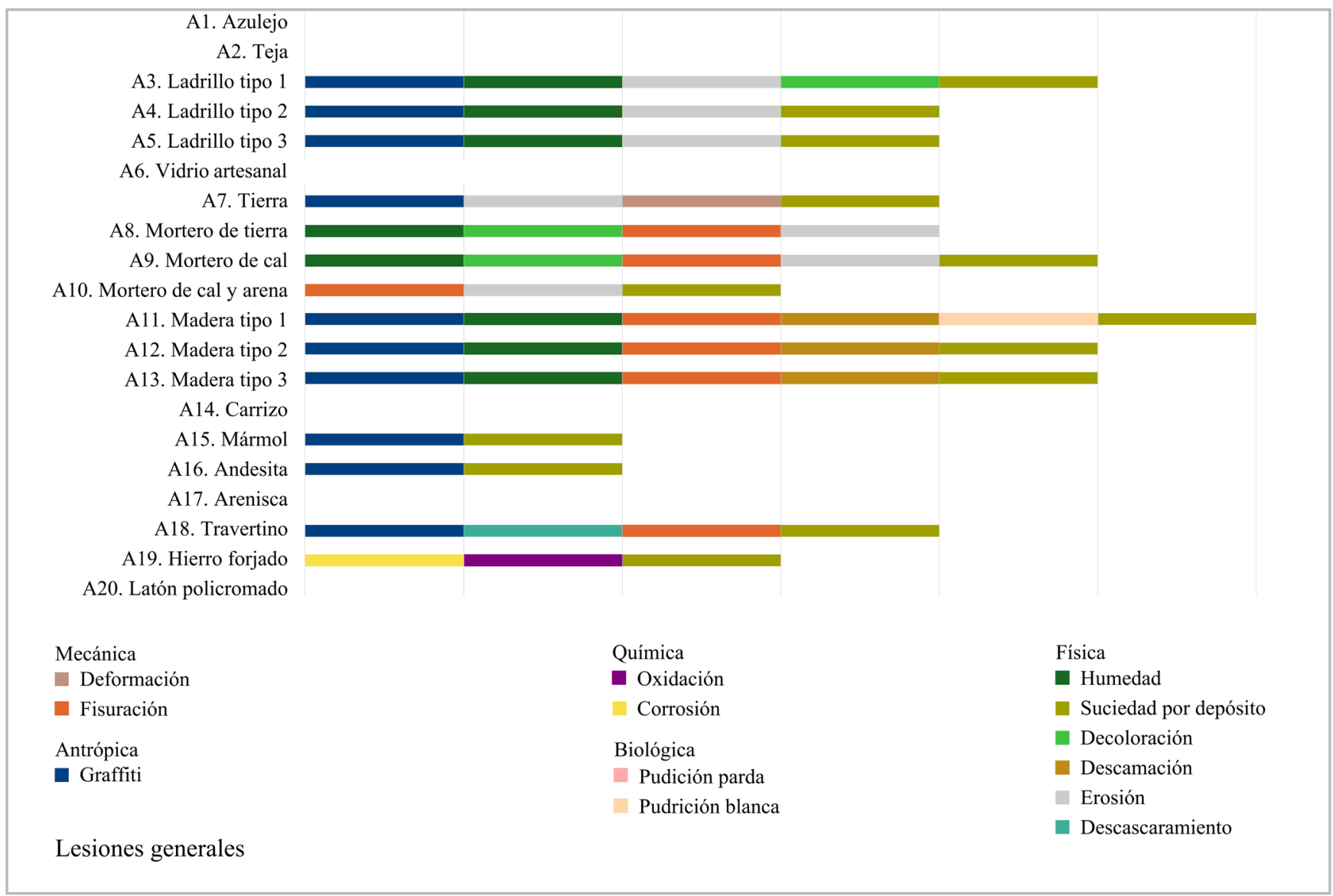

Figura 8.- Lesiones generales por material. Fuente y Elaboración: Autores.

Al considerar la EIA desde valoraciones parciales la premura de medidas de intervención disminuye. Un caso reporta incidencia en nivel Severo, mientras 3 en Atención Especial y 31 en Moderado [figura 9]. En los casos más frecuentes, coinciden la valoración patrimonial significativa e intensidad de uso. Asimismo, la adaptación con o sin proceso de intervención acentúa el detrimento, y por tanto el mal estado de conservación y vulnerabilidad a la acción de factores de deterioro. Este hecho ratifica que las condiciones particulares de los casos son concomitantes en sus afecciones; la naturaleza, acabado y exposición al medio, suponen un grupo de los factores de impacto, cuya acción sinérgica determina en máxime la condición patológica de cada material. De otro lado, si la valoración se produce por impacto acumulado y material [figura 10] el panorama es menos emergente aún, ya que solo el mortero de cal y cemento demanda Atención Especial; el resto de materiales se enmarcan en el nivel Moderado. 


\begin{tabular}{|c|c|c|c|c|c|c|c|c|c|c|c|c|c|c|c|c|c|c|}
\hline \multirow{3}{*}{ CASO } & \multicolumn{18}{|c|}{ Materiales } \\
\hline & \multicolumn{6}{|c|}{ Cerámicos, térreos y vítreos } & \multicolumn{3}{|c|}{ Morteros } & \multicolumn{3}{|c|}{ M. de origen vegetal } & \multicolumn{4}{|c|}{ Pétreos } & \multicolumn{2}{|c|}{ Metálicos } \\
\hline & A1 A2 & A3 & A4 & A5 & A6 & A7 & A8 & A9 & A10 & A11 & A12 & A13 A14 & A15 & A16 & A17 & A18 & A19 & A20 \\
\hline CBM05_CAT & & & & & & 44 & 38 & & & & & & & & & & & \\
\hline CBM01_CML & & 43 & & & & & & & & & & & & & & & & \\
\hline CBM02_CMG & & & & & & & 40 & & & 41 & & & & & & & & \\
\hline CBM03_CRC & & & & & & 41 & & & & & & & & & & & & \\
\hline CBM04_CVV & & 44 & & & & & & & & & & & & & & & & \\
\hline CSB01_CAA & & & & & & & & & & & & & & & & 42 & & \\
\hline CSB02_CC & & & & & & 49 & & & & 48 & & & & & & & & \\
\hline CSB03_CD & & & & & & & 44 & 43 & 46 & 46 & 46 & 47 & & & & 46 & & \\
\hline CSB04_CM & & & & & & & 44 & 44 & 47 & 43 & 43 & 43 & & & & 46 & & \\
\hline CSB05_CVD & & & & & & 44 & & & & & & & 44 & & & & & \\
\hline CPB01_CBI & & & & & & & 43 & & & & & & & & & 41 & & \\
\hline CPB02_CS & & & & 44 & & & & & & & & & & 39 & & & & \\
\hline CPB03_CA & & & & & & & 47 & & & 45 & & & & & & & & \\
\hline CL01_CAB & & & & 44 & & & & & & & & & & & & 48 & 43 & \\
\hline CL02_CAE & & & & & & & 44 & & & & & & & & & 42 & & \\
\hline CL03_CDO & & & & 44 & & & & & & & & & & & & & & \\
\hline CL04_CDA & & 43 & & & & & & & & & & & & & & 45 & & \\
\hline CL05_CGC & & 38 & 49 & & & & & & & & & & & & & 42 & 37 & \\
\hline CL06_CTO & & & 41 & 45 & & & & & & & & & & & & & & \\
\hline CL07_CUC & & 49 & & & & & & & & & & & & & & 43 & 52 & \\
\hline CPA01_CDA & & & & & & & 43 & & & & & & & & & & & \\
\hline CPA02_CVA & & & & & & & & & & & & & 46 & & & & & \\
\hline CPC01_CAM & & & & & & & & & 52 & 47 & & & & & & & & \\
\hline CPC02_CCR & & & & & & 46 & & & & & & & & & & & & \\
\hline CPC03_CCC & & 41 & & & & 45 & & & & & & & & & & & & \\
\hline CPC04_CTM & & & & & & & & & & & & & & 43 & & & & \\
\hline CPC05_CVA & & & & & & 43 & & & & & & & & & & & & \\
\hline CRMA01_CAO & & & & & & 46 & & & & & & & & & & & & \\
\hline CRMA02_CGMun & & 38 & & & & 51 & 53 & & & 39 & & & & & & & & \\
\hline CRMA03_CGM & & & & & & 42 & 46 & & & 47 & & & & & & & & \\
\hline CRMA04_CMD & & & & & & 40 & & & & 45 & & & & 40 & & & & \\
\hline CT01_CGMun & & & & & & 56 & & & & & & & & & & & 49 & \\
\hline AVDA01_CA & & & & 38 & & & & & & 45 & & & & & & & & \\
\hline AVDA02_MU & & 46 & & & & & & & & & & & & 45 & & & & \\
\hline AVDA03_CVM & & & & 40 & & & & & & & 42 & & & & & & & \\
\hline \multicolumn{19}{|c|}{ Simbología } \\
\hline Irrelevante & $0-25$ & & Mod & erado & & $25-5$ & 50 & & & vero & & $50-75$ & & & ítico & & & \\
\hline & & & & Atenció & ión es: & pecia & al: lín & ite ha & cia el & nivel ir & media & to superior & & & & & & \\
\hline
\end{tabular}

Tabla 2.- EIA por material. Fuente y Elaboración: Autores.

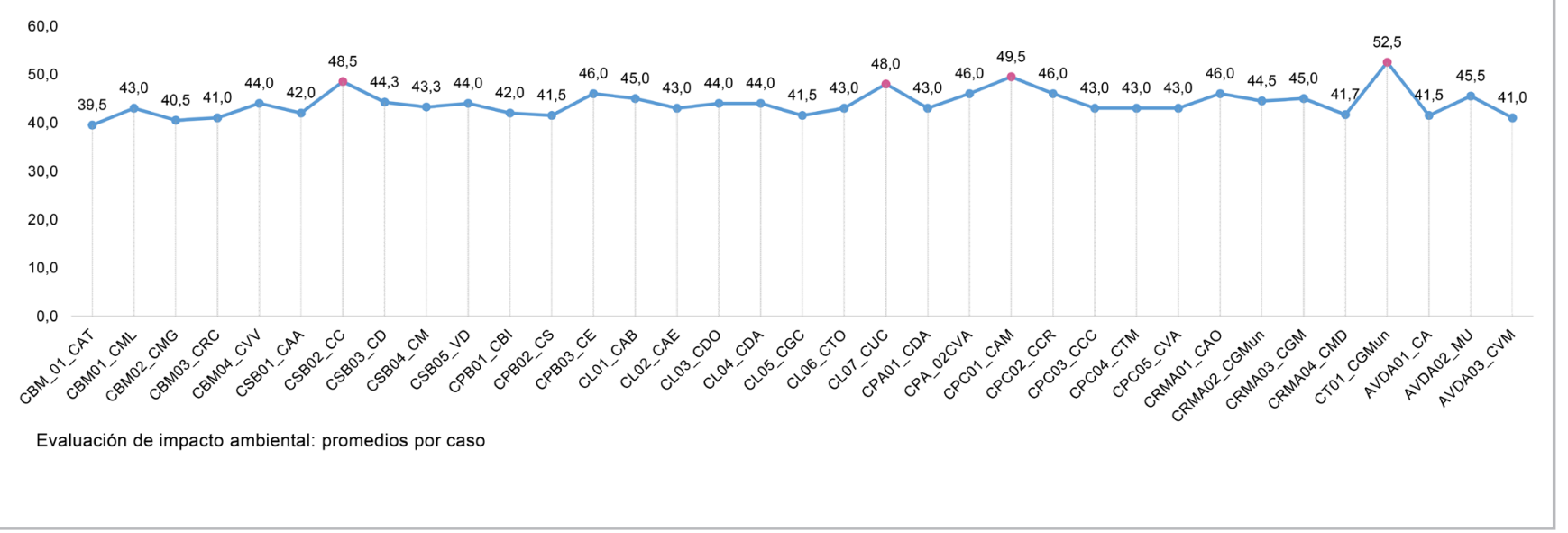

Figura 9.- EIA: promedios por caso. Fuente y Elaboración: Aguirre Ullauri 2019. 


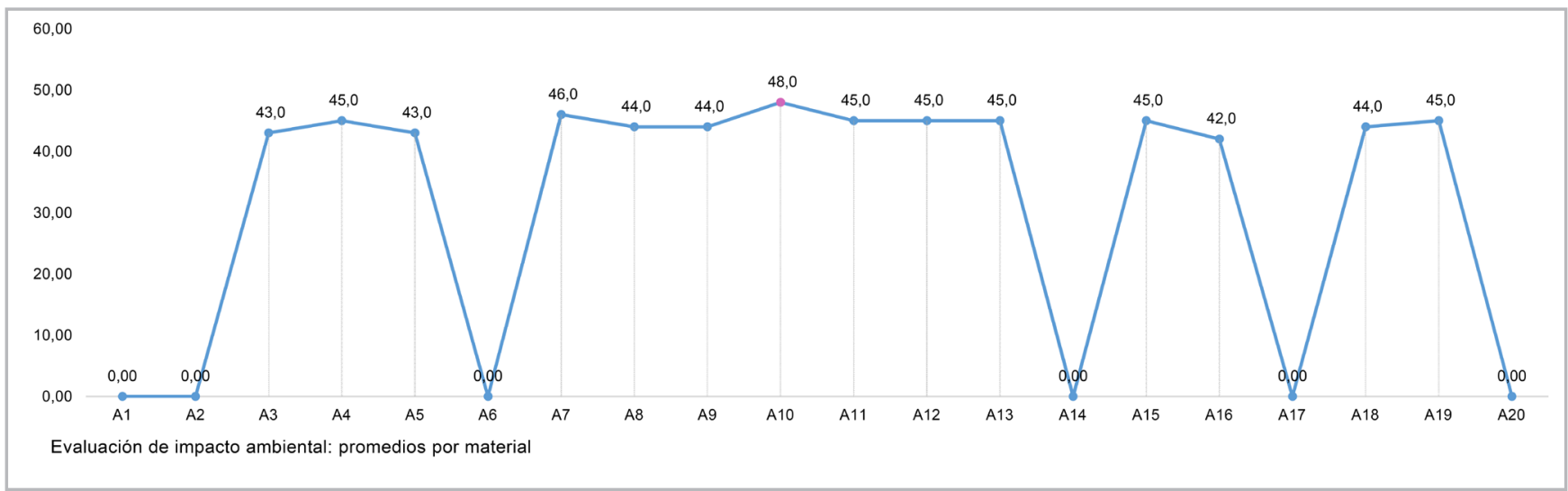

Figura 10.- ElA: promedios por material. Fuente y Elaboración: Aguirre Ullauri 2019.

Como contraparte del estudio patológico, y para su cualificación es indiscutible considerar que en contextos urbano - arquitectónicos deteriorados, los sentimientos de empoderamiento ciudadano disminuyen, contribuyendo a posicionar la vulnerabilidad de las preexistencias frente a la acción antropogénica destructora. Sectores como El Barrial Blanco o El Vado se definen como inseguros, insalubres y desordenados en el imaginario ciudadano. Por ello, la tipificación de lesiones y descripción de interacciones describen además los sentimientos de reconocimiento y estima ciudadana, articulados a visiones productivas y turísticas. En última instancia, el deterioro conforma un indicador de habitabilidad y de ello, la necesidad de propender hacia el buen vivir.

En este punto conviene la evaluación retrospectiva. En general, la distribución de los materiales de uso histórico no tiene relación directa, ya sea con el valor patrimonial vigente, a su vez con estilos y la ElA. Los materiales predominantes presentan impactos Moderados, aun cuando se han detectado múltiples afecciones, lo que ratificaría sus cualidades resistentes; por su parte el material más vulnerable, también reporta incidencia Moderada, aunque expone menores lesiones que los anteriores [figura 8], ya que su uso es menos frecuente en edificaciones al definirse como el material por excelencia de las vías del $\mathrm{CHC}$. Desde la singularidad de los casos analizados es advertible la continuidad en el nivel de afección de los materiales, pues las mayores variaciones, entre 15 y 16 puntos son escasas. Igual particular se evidencia al considerar cada edificación; el promedio oscila entre 4 y 6 puntos. Por ello, las condiciones descritas invitan a investigar sobre los materiales y sus condiciones intrínsecas a fin de discriminar los pormenores de su vulnerabilidad y/o resistencia.

Metodológicamente, la EIA es un planteamiento efectivo, aunque pueden generarse incordios, permite orientar la toma de decisiones priorizada a la hora de la aplicación normativa y estudios analíticos. Asimismo al considerar las variables a), b) y c) de la selección de casos en estricto orden, se evidencia que a) y c) podrían ser irrelevantes si b) presenta mayores efectos multiplicadores. Por ello, se asume la importancia de la ubicación de los casos y prevalece a) y 3 de b), es decir, la presencia de los materiales de interés. Insertando las condiciones acumuladas de la EIA asociadas al uso, tipo e intensidad, se conforma el primer escenario a nivel introductorio, emergente y corto plazo. A mediano y largo plazo tiene visión de constituirse como recurso para la conservación integral.

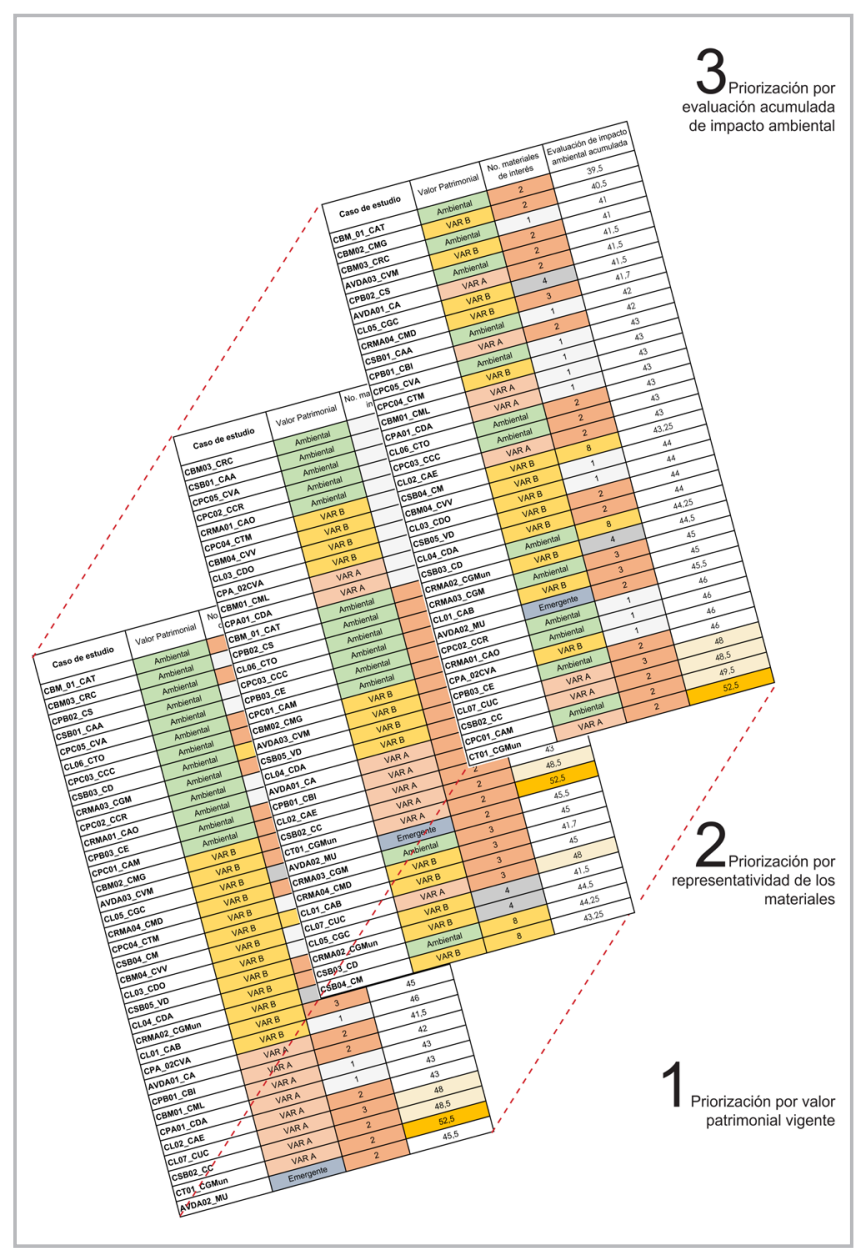

Figura 11.- Priorización de casos para el estudio analítico. Fuente y Elaboración: Aguirre Ullauri 2019. 


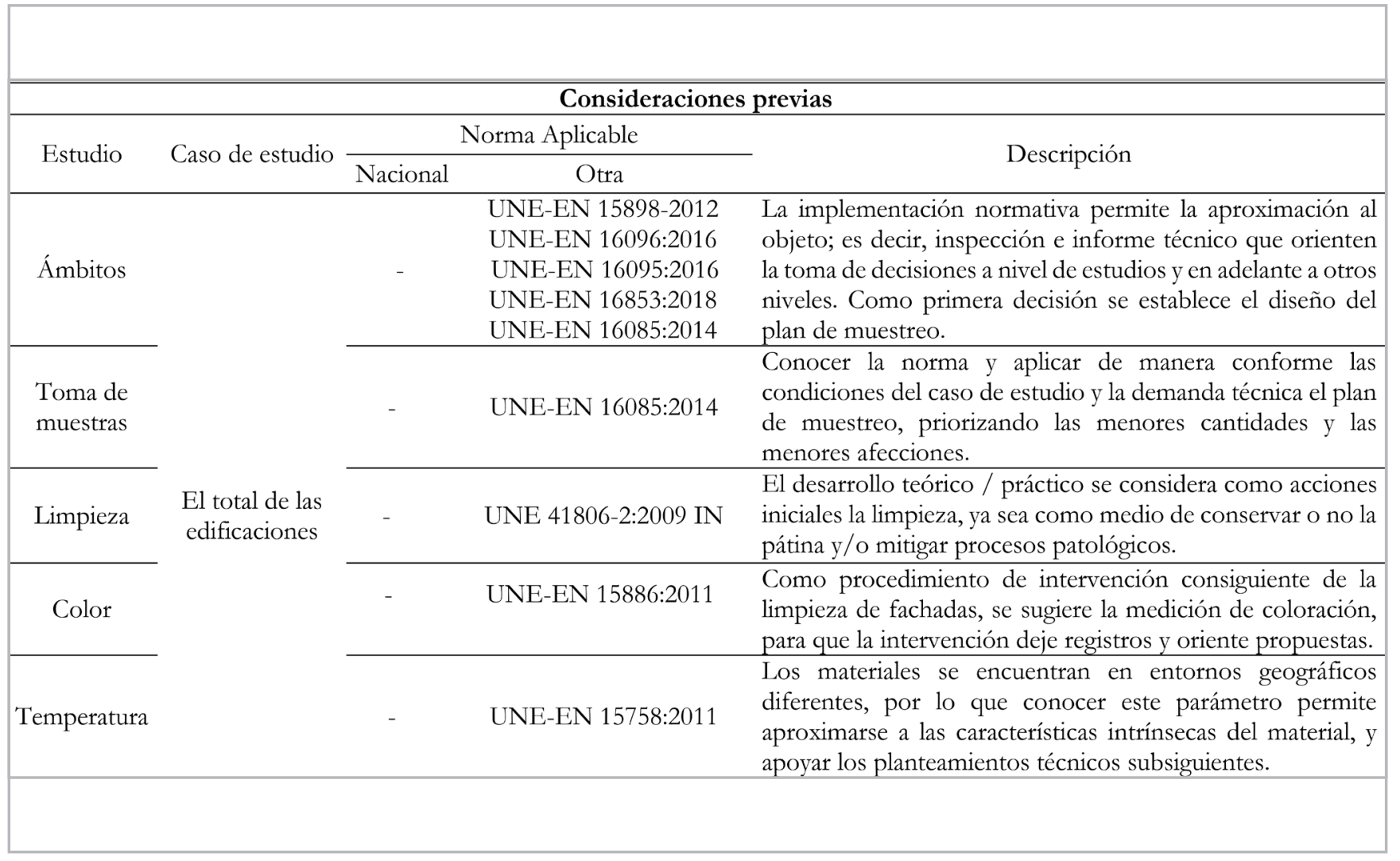

Tabla 3.- Consideraciones previas para la intervención en fachada. Fuente: Normas UNE. Elaboración: Castillo Carchipulla 2019

Finalmente, los estudios analíticos se enmarcan en la ElA y consideran que las prioridades de diseño de los edificios están conformadas por paradigmas y sistema de valores de la sociedad, la cultura y contexto dentro del cual emergen (Rapoport 1969; King 2004; Du Plessis 2012; Cole 2012). De esta manera la normativa da soporte técnico a valoraciones y procesos para intervenciones de edificios y fachadas patrimoniales.

Se parten de ensayos generales de identificación, hasta los específicos de caracterización [tabla 4]. Se recurre a las normas del Instituto Nacional de Normalización (INEN) que planifican, organizan, dirigen, controlan y evalúan los parámetros de la calidad, inocuidad y seguridad de los productos y servicios que se comercializan en el país, entre ellos los materiales de construcción. Estas normas se basan en otras provenientes de entidades como la American Society for testing and Materials (ASTM), la Asociación Española de Normalización y Certificación (AENOR) o el Comité Europeo de Normalización (CEN). Un ejemplo es el ensayo para determinar el límite líquido y límite plástico, que recurre a las normas ASTM D 4318 - 17 y NTE INEN 0691, pero la INEN recurre a la primera. Sin embargo, en otros casos sucede lo contrario.

Por estos hechos, y las consideraciones particulares de los materiales del patrimonio arquitectónico, como la mínima intervención, respeto por la autenticidad, integridad, entre otros, es necesario hacer un alcance mayor a la aplicabilidad de normativa internacional, ya sean ASTM, AENOR, CEN o semejantes. Por su parte, las normas INEN o NEC se descartan por su orientación a la obra nueva, carácter destructivo y constante referencia a normas internacionales. En este contexto, las normativas CEN del segmento español (UNE), francés (Commission 25-PEM o RILEM 1980) e italiano (UNI) se definen como las apropiadas. A su vez, a efectos propositivos se consideran las primeras, y define el escenario formado por dos momentos; el primero de Consideraciones Previas, y b) Propuesta de aplicación normativa internacional.

En el primer caso mientras las normas UNE-EN 16096, 16095, 16853 y 16085 definen el proceso. Las normas UNE-EN 15898 y 16515 deben considerarse por añadidura [Tablas 3]. En el segundo las normas aplicables son UNEEN $12407,14066,15886,13755,1936,12372,13161,1926$, 1925, 15801, 12370, 12371, 16455, 13919, 16682, 15803, 14581,16140 y 14158 . Ante este amplio panorama, pero por limitaciones técnicas, el espectro analítico se reduce, e incluye la Norma Peruana, ASTM y técnicas particulares factibles de aplicarse con mínimas variaciones técnicas. A efectos inferenciales se consideran los casos $\mathrm{CSBO}_{2}$ CC, CPC01_CAM, CL07_CUC, CSB05_VD y CT01_CG Mun, como la muestra significativa para desentrañar los particulares físicos, químicos y biológicos de los 14 materiales identificados en los 35 casos de estudio, y con ellos potencialmente el $98,69 \%$ de las fachadas de edificaciones patrimoniales del CHC. 


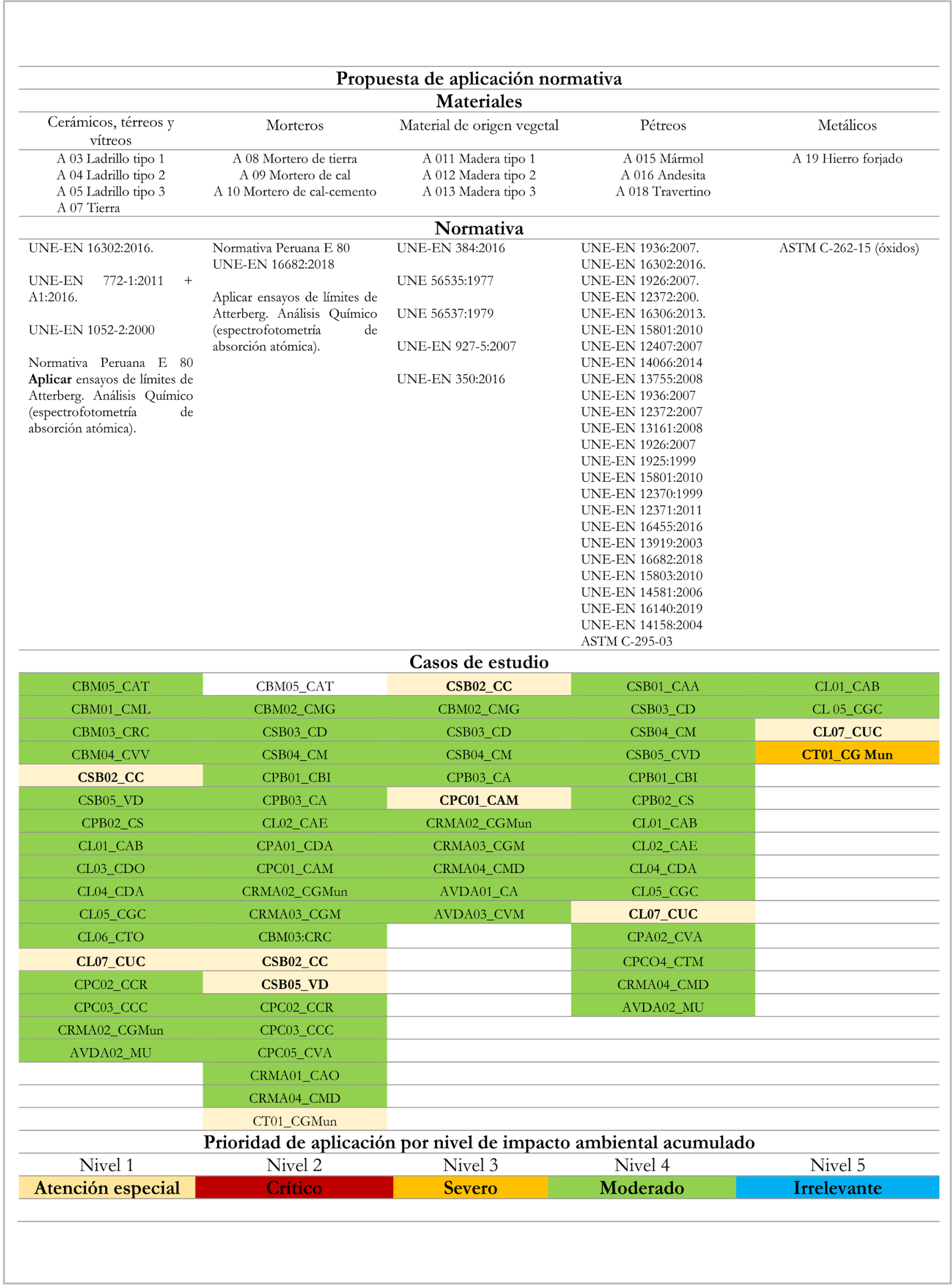

Tabla 4.- Normativa internacional para análisis de los materiales identificados en cada caso de estudio. Fuente: Normas UNE, Norma Peruana y ASTM. Elaboración: Castillo Carchipulla y Aguirre Ullauri 2019. 


\section{Conclusiones}

En el Centro Histórico de Cuenca por su singularidad temporal y cualidad de mosaico constructivo sobresalen tramos y edificaciones a partir a partir de la envoltura, como elemento artístico y estético por excelencia. Este reflejo del paso del tiempo ha colocado y descolocado estilos, tendencias y singularidades, a costa de la bondad constructiva primigenia que devela procesos de deterioro y describe nuevas dinámicas. No menos incidentes han sido los factores socio culturales, económicos y políticos, o el manteamiento e intensidad de uso, las características intrínsecas del material, la técnica y proceso constructivo como catalizadores de síntomas y cambios. Por ello, esta apariencia sobrevalorada define al bien patrimonial como resiliente.

A partir del diagnóstico patológico de los materiales en la fachada de edificios patrimoniales del $\mathrm{CHC}$ es factible colegir que, si bien el estado de conservación de los 14 materiales no llega a ser crítico, el deterioro es notable y potencial. Lo previo se consolida desde los ejercicios analíticos entre material, lesión patológica y evaluación ambiental, por ello la necesidad de ampliar su conocimiento, valoración y tratamiento técnico hacia niveles más complejos, si bien el planteamiento metodológico, conforma una ruta inicial, y el escenario de caracterización una propuesta.

La diversidad de lesiones patológicas identificadas en tipo y nivel de desarrollo elevan el entendimiento de la fachada o envolvente desde lo estético, hasta su desempeño como barrera ante los factores perjudiciales exteriores. Por ello, ha sido significativo evidenciar a través de 35 casos de estudio, la relación indisoluble entre arquitectura patrimonial, conservación y ambiente. De hecho, proyectar posibles vínculos con el declive salubre habitacional, resulta interesante, y seguro reunirá más adeptos que el diagnóstico patológico exclusivamente arquitectónico. Por su parte, las políticas locales aplicadas a la prevención de incidencias no han trascendido a las particularidades del contexto histórico enfocándose en la salud pública, aunque con limitaciones, y relegando el ámbito patrimonial, habitacional y de recursos del territorio. En ambos casos, urge debatir y desarrollar instrumentos de gestión del hábitat patrimonial.

\section{Notas}

[1] Organización no gubernamental que operó en Ecuador entre 1977 y 2012. Se orientaba hacia el cuidado de la naturaleza a partir de proyectos de gestión de residuos sólidos, biodiversidad, áreas protegidas, desarrollo sostenible y más. Actualmente mantiene actividad en Colombia, Bolivia y Europa.

[2] Define al periodo entre 1890 y 1940 cuando la ciudad experimenta incrementos sustanciales de producción cuyos excedentes se revierten en la modernización. La arquitectura juega un rol renovador de la imagen a través de la influencia francesa, propia de la vinculación al mercado mundial.

[3] Actualmente se trabaja a partir de la aplicación del método de lectura estratigráfica para determinar a través de las acciones físicas y las nociones de anteroposterioridad, las relaciones de deterioro existentes en los materiales de interés.

[4] La realización de cuatro inventarios ha derivado en tres esquemas de clasificación. El básico o por grupos, 1, por escala y 2, de arquitectura civil (Washima Tola 2014: 85-87) según el inventario de 1978; el intermedio del año 99 o de categorías, VHIAR (Valor Histórico Arquitectónico) I para inmuebles de valor emergente, uso colectivo y con características que sobresalen en la trama urbana; VHIAR II para inmuebles con características sobresalientes y de uso residencial; y VHIAR III, para aquellos que presentan rasgos notables que resaltan la calidad del contexto urbano (Jaramillo $\mathrm{s} / \mathrm{f}:$ 10-11). Finalmente se encuentran las del 2009 y recogidas en la ordenanza vigente y utilizadas en la presente investigación.

[5] Según la ordenanza vigente al primer caso se corresponden las edificaciones que cumplen un rol constitutivo en la morfología del tramo, de la manzana o del área en la que se insertan por sus características estéticas, históricas, o por su significación social; el segundo refiere a edificaciones cuyo rol es el de consolidar un tejido coherente con la estética de la ciudad o el área en la que se ubican y pueden estar enriquecidas por atributos históricos o de significados importantes para la comunidad local. Desde el punto de vista de su organización espacial expresan con claridad formas de vida que reflejan la cultura y el uso del espacio de la comunidad. En el último caso, las edificaciones que se caracterizan por permitir y fortalecer una legibilidad coherente de la ciudad o del área en la que se ubican. Son edificaciones cuyas características estéticas, históricas o de escala no sobresalen de una manera especial, cumpliendo un rol complementario en una lectura global del barrio o de la ciudad. Sus características materiales, la tecnología utilizada para su construcción y las soluciones espaciales reflejan fuertemente la expresión de la cultura popular.

[6] Dichos estudios no se han emprendido en el medio pese a la representatividad de los materiales en la arquitectura histórica e influencia en la moderna y contemporánea. En el caso de la madera el aporte más reciente hace referencia al inventario de especies madereras para indicar que aquellas comunes de encontrar son; Mara, Motilón, Eucalipto, Capulí, Nogal, Colorado, Cedro, Laurel, Seique, Romerillo y Pino (Reyes \& Zaruma 2017); sin embargo, no diferencian entre nativas e introducidas. El Pino presente en el país desde 1850 (Farley 2008), o el Eucalipto, a partir 1865 (Acosta Solís 1949). Esto mantiene la incertidumbre de la utilización de ciertas especies y cómo su presencia representa valores agregados. Por su parte al hablar del ladrillo es preciso indicar que su producción se mantiene artesanal, y su estudio alcanza en máxima instancia los productos contemporáneos desde las características físicas y mecánicas.

[7] La problemática se enmarca en la disponibilidad de instrumentación específica conforme el estado de los laboratorios locales, por lo que la actividad se desplazaría a entidades como 
la Escuela Politécnica del Litoral (Guayaquil), la Universidad Regional Amazónica (Tena) o el Laboratorio de Investigación y Análisis del INPC (Quito).

\section{Referencias}

ACHIG BALAREZO, M. C., ZÚÑIGA LÓPEZ, M., BALEN, K. V., y ABAD RODAS, M. L. (2013). "Sistema de registro de daños para determinar el estado constructivo en muros de adobe". Maskana, Vol. 4, No. 2. 71-84. https://doi.org/10.18537/mskn.04.02.06

ACHIG BALAREZO, M., ZÚÑIGA, M., GARCÍA, G., AGUIRRE, A., GALÁN, N. BARSALLO, G. y BRIONES, J. (2016). "Altas de Daños. Edificaciones Patrimoniales de Cuenca". Proyecto vlirCPM. Universidad de Cuenca.

ACOSTA SOLÍS, M. (1949). El Eucalipto en el Ecuador. Instituto Ecuatoriano de Ciencias Naturales. Departamento Forestal del Ecuador. Quito: Editorial Ecuador.

AGUIRRE ULLAURI, M. y SANZ ARAUZ, D. (2017). “Caracterización de materiales históricos para el conocimiento y la conservación del Patrimonio Arquitectónico: revisión y proyección en el caso de Cuenca (Ecuador)". En Traversa, L. (com.). Actas del V Congreso Iberoamericano y XIII Jornada Técnica de Restauración y Conservación del Patrimonio. La Plata: Laboratorio de Entrenamiento Multidisciplinario y Tecnológico, 48 - 64.

AGUIRRE ULLAURI, M., SOLANO PELÁEZ, J. GARCÍA C., A., LÓPEZ L.D., CARRIÓN C, P., YAMUNAQUÉ A., L. (2018). “Evaluación del impacto ambiental en la Arquitectura Patrimonial a través de la aplicación de la Matriz de Leopold como un posible sistema de monitoreo interdisciplinar". ASRI. Arte y Sociedad. Revista de Investigación. 14, 17 - 34.

AGUIRRE ULLAURI, M., SOLANO PELÁEZ, J., SEGARRA VILLACÍS, C. y CARRIÓN CABRERA, P. (2018). "El impacto ambiental sobre los materiales históricos: Evaluación de Niveles de Incidencia en el caso de Cuenca (Ecuador)". En Quinto Congreso Internacional de Medio Ambiente Construido y Desarrollo Sustentable. MACDES 2018. La Habana (Cuba).

ALBERTALLI, M. (2009). "Blanco sobre negro. La dimensión artística de la superficie" en A. M. Rigotti, S. Pampinella (ed.) Una cosa de vanguardia: Hacia una Arquitectura (Rosario, UNR ed.), 6881.

BALARAS C., DROUTSA K., DASKALI, E., KONTOYIANNIDIS, S. (2005). "Deterioration of European apartment buildings". Energy Build, 37, 515-27. https://doi.org/10.1016/j. enbuild.2004.09.010

CARDOSO, F. (2012). Metodología de Manuales de Conservación Preventiva. Universidad de Cuenca. Ecuador.

CARDOSO, F. (ed.) (2017). Propuesta de inscripción del Centro Histórico de Cuenca, Ecuador en la lista de patrimonio mundial. Edición Comentada. Cuenca: Grafisum.
CONSEJO CANTONAL DE CUENCA. (2010). Ordenanza para la Gestión y Conservación de las Áreas Históricas y Patrimoniales del Cantón Cuenca. Cuenca. Ecuador.

COLE, R. J. (2012). "Transitioning from green to regenerative design", En Building Research \& Information, 40(1), 39-53. https:// doi.org/10.1080/09613218.2011.610608

DE JONGH, S., VAN WIJNENDDAELE, M., CARDOSO y VAN BALEN. K, (2009). Damage Atlas of historial Cuenca, Ecuador. Damage patterns found in the (mainly earthen) building materials. Katholieke Universities Leuven. VLIR - IUOS. Consultado en https://es.slideshare.net/mvanwijnendaele/damage-atlashistorical-cuenca (2017-10-18).

DU PLESSIS, C. (2012). "Towards a regenerative paradigm for the built environment". Building Research \& Information, 40(1), 7-22. https://doi.org/10.1080/09613218.2012.628548

DOUGLAS-JONES, R., HUGHES, J. y YARROW, T. (2016). "Science, value and material decay in the conservations of historic environments". Journal of Cultural Heritage, 21, 823 - 833. https:// doi.org/10.1016/j.culher.2016.03.007

ESPINOZA, P., CALLE, M. (2002). La citécuencana:el afrancesamiento de Cuenca en la época republicana (1860-1940) (Tesis de Pregrado). Universidad de Cuenca, Ecuador.

FARLEY, K. (2008). "Grasslands to Tree Plantations: Forest Transition in the Andes of Ecuador". Annals of the Association of American Geographers, 97:4, 755-771. https://doi.org/10.1111/ j.1467-8306.2007.00581.x

GAD DE CUENCA (2010). Pliegos para la contratación de la Elaboración del Estudio del Plan de Conservación incluido en el Proyecto Plan Especial de Centro Histórico - Segunda Etapa. Secretaría General de Planeamiento. Dirección de Áreas Históricas y Patrimoniales. Cuenca.

HERAS, V. (2015). “Cuenca, quince años como patrimonio mundial: evaluación de procesos de documentación y monitoreo". ESTOA. Revista de la Facultad de Arquitectura de la Universidad de Cuenca. 6: 27-35. https://doi.org/10.18537/est.v004.n006.06

INSTITUTO NACIONAL DE PATRIMONIO CULTURA -INPC (2011). Guía de medidas preventivas para amenaza sísmica, seguridad, protección y manejo de bienes culturales. Serie Normativas y Directrices. Quito: Ediecuatorial.

ILUSTRE CONSEJO CANTONAL (2000). Reglamento para el uso del color y materiales en las edificaciones del Centro Histórico. Cuenca.

JARAMILLO PAREDES, D. (s/f). Estado de los inventarios del patrimonio cultural edificado en la ciudad de Cuenca-Ecuador, Cuenca-Ecuador, Universidad de Cuenca. (Manuscrito Inédito).

KING, A. (2004). Spaces of Global Cultures: Architecture Urbanism Identity, Routledge, London. 
LORENZ, K. (2004). Los ocho pecados mortales de la humanidad civilizada. Buenos Aires: Gráficas Guadua. S. A.

MATARAN ANGUELA, J.A. (1996). Normativa sobre el patrimonio histórico cultural. Madrid: EGRAF, S.A.

MUÑOZ, P. (2015). Arquitectura popular en Azuay y Cañar. 19771978. Cuenca: CIDAP.

ORELLANA SAMANIEGO, M. SELLERS WALDEN, CH., MARTÍNEZ GAVILÁNEZ, J. (2017). "Índice de calidad ambiental urbana de Cuenca". Universidad y Verdad. 73, 66 - 77.

QUEROL, M. (2010). Manual de gestión del patrimonio cultural. Madrid: Ediciones Akal.

RAPOPORT, A. (1969). House Form and Culture, Prentice. Hall, Englewood Cliffs, NJ. https://doi.org/10.1126/ science.167.3916.274

REYES, J., ZARUMA, L. (2017). Patologías de la madera. Identificación y solución de las principales patologías presentes en estructuras de madera de edificaciones patrimoniales localizadas en el Centro Histórico de Cuenca - Ecuador. (Tesis de pregrado). Universidad de Cuenca. Cuenca.

RODAS V. C., ASTUDILLO, S. y RODAS, J. (2018). “En busca del equilibrio entre la transformación y conservación. Inserción de proyectos contemporáneos en ciudades Patrimonio de la Humanidad. El caso de Cuenca, Ecuador". ASRI: Arte y Sociedad, 14, $215-235$.

RODRIGUES, M., TEIXEIRA, J., CARDOSO, J. (2011). "Building envelope anomalies: A visual survey methodology" Constructions and Building Materials, 25, 2741 - 2750. https://doi.org/10.1016/j. conbuildmat.2010.12.029

RODRíGUEZ, V. (coord.). (2004). Manual de Patología de la Edificación. Tomo I, II y III. Departamento de Tecnología de la Edificación. Universidad Politécnica de Madrid.

RUEDA, E. (2014). "Propuesta de mapa de riesgos de bienes patrimoniales inmuebles expuestos a amenazas de origen natural", Enfoque UTE. 5(1), 30-48.

SAHADY VILLANUEVA, A., GALLARDO GASTELO, F. (2009). "En edificios de ayer, funciones de hoy. La vivienda: una constante histórica". Revista INVI, 17(45), 69 - 81.

VAN BALEN, K., VERSTRYNGE, E. (eds). (2016). Structural Analysis of Historical Constructions. Anamnesis, diagnosis, therapy, controls. Actas y Monografías de Ingeniería, Agua y Ciencias de la Tierra. Londres: Taylor \& Francis Group.

WASHIMA TOLA, S. (2014). El concepto de intervención en la normatividad para la conservación patrimonial en CuencaEcuador, (Tesis de Maestría). Instituto Nacional de Antropología e Historia. México.

\section{Autor/es}

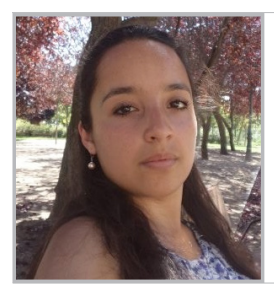

\section{María del Cisne Aguirre Ullauri}

cisne2222@hotmail.com - maguirreu@ ucacue.edu.ec

Universidad Politécnica de Madrid.

Universidad Católica de Cuenca

Arquitecta por la Universidad de Cuenca (2010), Máster Universitario en Conservación y Restauración del Patrimonio Arquitectónico (2014) y Estudiante del Programa de Doctorado en Patrimonio Arquitectónico por la Universidad Politécnica de Madrid (2015-2020). Docente Titular Auxiliar y Coordinadora del Centro de Investigación de Ingeniería, Industria, Construcción y TIC en la Universidad Católica de Cuenca (Ecuador). Directora del Proyecto de Investigación Los materiales en el estudio histórico - constructivo - ambiental de los conjuntos históricos. El caso de Cuenca., desde el 2017. Miembro de los Grupos de Investigación CAT -Ciudad, Ambiente y Tecnología- y C\&D -Ciencia \& Diversidadde la Universidad Católica de Cuenca (2017 y 2019), la Red Ecuatoriana de Mujeres Científicas -REMCl- (2017), y la Organization for Women in Science for the Developing World -OSDW- (2018).

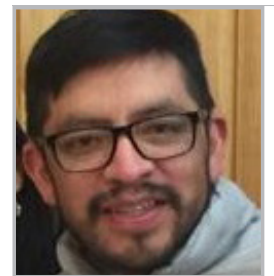

Edison Maximiliano Castillo Carchipulla

em.castillo@alumnos.upm.es Universidad Politécnica de Madrid Universidad Católica de Cuenca

Doctorando del Programa en Innovación Tecnológica en Edificación en la Universidad Politécnica de Madrid. Docente Ocasional de la Universidad de Cuenca (Ecuador). Miembro del American Concrete Institute (ACl) capítulo Ecuador. Director del Laboratorio de Aplicaciones Constructivas y Bioclimáticas de la Facultad de Arquitectura y Urbanismo de la Universidad de Cuenca (Ecuador). Participación con un grupo de estudiantes en el concurso ECO Concrete Quebec (Canadá) en marzo 2018, organizado por el $\mathrm{ACl}$, con el objetivo de promover la idea del desempeño ambiental en el diseño de mezclas de concreto, obteniendo el 2 do premio. Por su parte, en el laboratorio se realizan diversas actividades de investigación que corresponden al análisis de materiales (hormigón y morteros) y sus aplicaciones a nivel local.

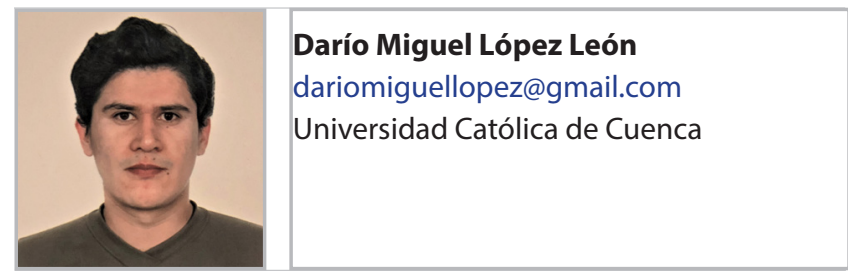

Arquitecto por la Universidad Católica de Cuenca (2019). Durante los últimos años de preparación profesional se ha vinculado a proyectos investigativos como: Los materiales en el estudio histórico-constructivo - ambiental de los conjuntos históricos. El 
caso de Cuenca, y Prefabricación de elementos de mampostería con fibra de totora. Como investigador independiente ha desarrollado indagaciones de carácter científico en el campo de la conservación patrimonial e innovación de materiales destinados a la construcción.

Artículo enviado el 24/07/2019 Artículo aceptado el 22/02/2020

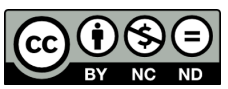

https://doi.org/10.37558/gec.v17i1.680 